\title{
Dynamical non-axisymmetric instabilities in rotating relativistic stars
}

\author{
Gian Mario Manca ${ }^{1,2}$, Luca Baiotti ${ }^{3}$, Roberto De Pietri ${ }^{1}$ \\ and Luciano Rezzolla ${ }^{3,4}$ \\ ${ }^{1}$ Dipartimento di Fisica, Università di Parma and INFN, Parma, Italy \\ ${ }^{2}$ Max-Planck-Institut für Astrophysik, Garching, Germany \\ ${ }^{3}$ Max-Planck-Institut für Gravitationsphysik, Albert-Einstein-Institut, Golm, Germany \\ ${ }^{4}$ Department of Physics and Astronomy, Louisiana State University, Baton Rouge, LA, USA
}

Received 20 April 2007, in final form 4 May 2007

Published 30 May 2007

Online at stacks.iop.org/CQG/24/S171

\begin{abstract}
We present new results on dynamical instabilities in rapidly rotating relativistic stars. In particular, using numerical simulations in full general relativity, we analyse the effects that the stellar compactness has on the threshold for the onset of the dynamical bar-mode instability, as well as on the appearance of other dynamical instabilities. By using an extrapolation technique developed and tested in our previous study (Baiotti L et al 2007 Phys. Rev. D 75 044023), we explicitly determine the threshold for a wide range of compactnesses using four sequences of models of constant baryonic mass comprising a total of 59 stellar models. Our calculation of the threshold is in good agreement with the Newtonian prediction and improves the previous post-Newtonian estimates. In addition, we find that for stars with sufficiently large mass and compactness, the $m=3$ deformation is the fastest growing one. For all of the models considered, the non-axisymmetric instability is suppressed on a dynamical timescale with an $m=1$ deformation dominating the final stages of the instability. These results, together with those presented in Baiotti L et al (2007 Phys. Rev. D 75 044023), suggest that an $m=1$ deformation represents a general and latetime feature of non-axisymmetric dynamical instabilities both in full general relativity and in Newtonian gravity.
\end{abstract}

PACS numbers: 04.25.Dm, 04.30.Db, 04.40.Dg, 95.30.Lz, 95.30.Sf, 97.60.Jd

(Some figures in this article are in colour only in the electronic version)

\section{Introduction}

Non-axisymmetric deformations of rapidly rotating self-gravitating bodies are rather generic phenomena in nature and could appear in a variety of astrophysical scenarios such as stellar core collapse [2], accretion-induced collapse of white dwarfs [3] or the merger of two neutron 
stars [4]. Over the years a considerable amount of work has been devoted to the search for unstable deformations that, starting from a quasi-axisymmetric stellar configuration, would lead to the formation of highly deformed rotating massive objects (see [1] for a detailed list of references). One of the main reasons behind this interest is that such deformations would lead to an intense emission of high-frequency gravitational waves (i.e., in the $\mathrm{kHz}$ range) which is potentially detectable by ground-based detectors such as LIGO, GEO, Virgo or the planned resonant detector such as DUAL [5].

Despite such extensive studies, various questions about the dynamics of the nonaxisymmetric deformation of rapidly rotating self-gravitating bodies are not yet completely clarified. Among the most important questions that have been addressed only rather recently it is worthwhile to recall the following: (i) how long do these deformations survive once they reach their maximum amplitude? (ii) how large is the energy emitted in gravitational waves? (iii) which physical phenomena determine the shortest damping timescale and impress a signature on the emitted signal? (iv) what is the effect of the stellar compactness $M / R_{e}$, where $M$ and $R_{e}$ are the stellar mass and the proper equatorial radius, respectively, on the dynamics of the instability and on the threshold for its onset?

While points (i)-(iii) were first addressed in [1] (hereafter paper I), here we concentrate on providing an answer to question (iv) supplying new information on the general properties of the dynamical instability in a very large class of stellar models that are characterized by differential rotation and by high compactness and that are members of four sequences of models with constant baryonic mass.

The main result obtained is that any non-axisymmetric deformation that develops in our models is damped over a dynamical timescale, through pure inviscid hydrodynamical nonlinear phenomena. Moreover, for all the models that develop dynamical instabilities, the $m=1$ deformation eventually becomes the dominant one irrespective of whether the models are above or below the threshold for the development of the bar-mode instability (see also paper I). This evidence is consistent with the simulations performed in Newtonian gravity by $\mathrm{Ou}$ and Tohline [6] for stars with a very-low $\beta$ (where $\beta \equiv T /|W|$ is the ratio between the rotational kinetic energy $T$ and the gravitational binding energy $W$ ), thus suggesting that this may be a general feature of this type of dynamical instability. In addition, we show that, in a region of high stellar compactness, other instabilities, such as one having an $m=3$ deformation, can develop. Finally, adopting an extrapolation technique developed and tested in paper I, we determine the threshold $\beta_{c}$ for the onset of the bar-mode instability for all of the sequences considered, thus determining accurately its dependence on the stellar compactness.

The paper is organized as follows. In section 2 we briefly describe the initial data chosen and the numerical techniques employed for their evolution, while in section 3 we review the tools used in the analysis of the data. Section 4 collects our results and there we first discuss the threshold of the bar-mode instability, its persistence and eventually the onset of higher-mode dynamical instabilities. Finally, section 5 contains our conclusions and the goals of our future research. Hereafter we use a space-like signature $(-,+,+,+)$, with Greek indices running from 0 to 3 , Latin indices from 1 to 3 and the standard convention for the summation over repeated indices. Unless explicitly stated, all the quantities are expressed in units in which $c=G=M_{\odot}=1$.

\section{Initial data and numerical evolution method}

Our simulations involve the numerical solution in three spatial dimensions (3D) of the full set of Einstein equations coupled to those of a perfect-fluid matter

$$
G_{\mu \nu}=8 \pi T_{\mu \nu},
$$




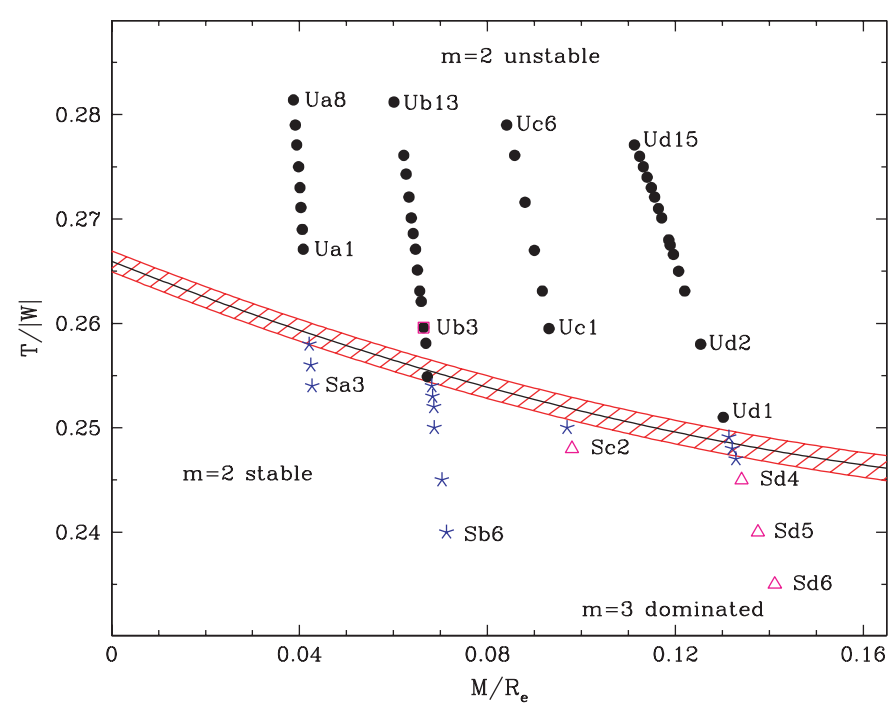

Figure 1. Position on the $\left(M / R_{e}, \beta\right)$ plane of the considered stellar models. Indicated respectively with stars and filled circles are the $m=2$-stable and $m=2$-unstable models belonging to the four sequences of constant rest mass. Triangles refer instead to models where the $m=3$ deformation is the fastest growing one. Indicated with a solid line is the threshold of the bar-mode instability, while the dashed region represents the region of our estimated error-bars.

where

$$
T^{\mu \nu}=\rho\left(1+\epsilon+\frac{p}{\rho}\right) u^{\mu} u^{v}+p g^{\mu \nu},
$$

and $u^{\mu}$ is the fluid 4-velocity, $p$ is the fluid pressure, $\epsilon$ the specific internal energy and $\rho$ the rest-mass density, so that $e=\rho(1+\epsilon)$ is the energy density in the rest frame of the fluid. The evolution of the spacetime must be supplemented by the evolution of the relativistic hydrodynamics equations: the conservation laws for the energy-momentum tensor $\nabla_{\mu} T^{\mu \nu}=0$ and the baryon number $\nabla_{\mu}\left(\rho u^{\mu}\right)=0$, complemented with an equation of state (EOS) of type $p=p(\rho, \epsilon)$.

The initial data for our simulations are computed as stationary equilibrium solutions for axisymmetric and rapidly rotating relativistic stars in polar coordinates [7]. In generating these equilibrium models the metric describing an axisymmetric relativistic star is assumed to have the form

$$
\mathrm{d} s^{2}=-\mathrm{e}^{\mu+v} \mathrm{~d} t^{2}+\mathrm{e}^{\mu-v} r^{2} \sin ^{2} \theta(\mathrm{d} \phi-\omega \mathrm{d} t)^{2}+\mathrm{e}^{2 \xi}\left(\mathrm{d} r^{2}+r^{2} \mathrm{~d} \theta^{2}\right),
$$

where $\mu, v, \omega$ and $\xi$ are space-dependent metric functions. As in paper I, we assume the matter to be characterized by a non-uniform angular-velocity distribution of the form

$$
\Omega_{c}-\Omega=\frac{r_{e}^{2}}{\hat{A}^{2}}\left[\frac{(\Omega-\omega) r^{2} \sin ^{2} \theta \mathrm{e}^{-2 v}}{1-(\Omega-\omega)^{2} r^{2} \sin ^{2} \theta \mathrm{e}^{-2 v}}\right],
$$

where $r_{e}$ is the coordinate equatorial stellar radius and the coefficient $\hat{A}$ is a measure of the degree of differential rotation, which we set to $\hat{A}=1$. All the equilibrium models considered here have been calculated using the relativistic polytropic EOS $\left(p=K \rho^{\Gamma}\right)$ with $K=100$ and $\Gamma=2$ and are members of four sequences having a constant rest mass $M_{*}$ equal to $1.0 M_{\odot}, 1.51 M_{\odot}, 2 M_{\odot}$ and $2.5 M_{\odot}$, respectively. The main properties of the four sequences are reported in tables $1-4$. The baryonic mass $M_{*}$, the gravitational mass $M$, the angular 
Table 1. Main properties of the stellar models of the sequence with $M_{*}=1 M_{\odot}$. Starting from the left: the name of the simulation, the compactness $M / R_{e}$, the instability parameter $\beta$, the central rest-mass density $\rho_{c}$, the ratio between the polar and the equatorial coordinate radii $r_{p} / r_{e}$, the proper equatorial radius $R_{e}$, the gravitational mass $M$, the total angular momentum $J$ divided by the square of the gravitational mass, the rotational periods at the axis $P_{a}$ and at the equator $P_{e}$. The initial letter in the model's name indicates whether it is an unstable (U) or a stable (S) configuration.

\begin{tabular}{llllllllll}
\hline Mod & $M / R_{e}$ & $\beta$ & $\rho_{c}\left(10^{-4}\right)$ & $r_{p} / r_{e}$ & $R_{e}$ & $M$ & $J / M^{2}$ & $P_{a}(\mathrm{~ms})$ & $P_{e}(\mathrm{~ms})$ \\
\hline Ua8 & 0.0387 & 0.2814 & 0.4369 & 0.21044 & 25.37 & 0.982 & 2.142 & 2.283 & 4.945 \\
Ua7 & 0.0391 & 0.2790 & 0.5662 & 0.23177 & 25.04 & 0.980 & 2.098 & 2.207 & 4.791 \\
Ua6 & 0.0394 & 0.2771 & 0.6429 & 0.24288 & 24.83 & 0.979 & 2.070 & 2.164 & 4.702 \\
Ua5 & 0.0398 & 0.2750 & 0.7087 & 0.25173 & 24.63 & 0.979 & 2.044 & 2.127 & 4.626 \\
Ua4 & 0.0401 & 0.2730 & 0.7672 & 0.25918 & 24.45 & 0.980 & 2.021 & 2.094 & 4.559 \\
Ua3 & 0.0403 & 0.2711 & 0.8168 & 0.26550 & 24.29 & 0.978 & 2.001 & 2.068 & 4.506 \\
Ua2 & 0.0406 & 0.2690 & 0.8668 & 0.27149 & 24.11 & 0.979 & 1.979 & 2.040 & 4.449 \\
Ua1 & 0.0408 & 0.2671 & 0.9107 & 0.27684 & 23.95 & 0.978 & 1.960 & 2.017 & 4.402 \\
Sa1 & 0.0421 & 0.2580 & 1.0907 & 0.29807 & 23.22 & 0.977 & 1.878 & 1.922 & 4.209 \\
Sa2 & 0.0424 & 0.2560 & 1.1270 & 0.30236 & 23.05 & 0.977 & 1.861 & 1.904 & 4.172 \\
Sa3 & 0.0427 & 0.2540 & 1.1619 & 0.30655 & 22.89 & 0.977 & 1.844 & 1.886 & 4.135 \\
\hline
\end{tabular}

Table 2. Same quantities as in table 1 for the sequence of models with $M_{*}=1.51 M_{\odot}$.

\begin{tabular}{llllllllll}
\hline Mod & $M / R_{e}$ & $\beta$ & $\rho_{c}\left(10^{-4}\right)$ & $r_{p} / r_{e}$ & $R_{e}$ & $M$ & $J / M^{2}$ & $P_{a}(\mathrm{~ms})$ & $P_{e}(\mathrm{~ms})$ \\
\hline Ub13 & 0.0601 & 0.2812 & 0.5990 & 0.20012 & 24.31 & 1.462 & 1.753 & 1.723 & 3.910 \\
Ub12 & 0.0622 & 0.2761 & 0.9938 & 0.24151 & 23.52 & 1.462 & 1.679 & 1.599 & 3.655 \\
Ub11 & 0.0626 & 0.2743 & 1.0920 & 0.25012 & 23.31 & 1.460 & 1.660 & 1.572 & 3.598 \\
Ub10 & 0.0633 & 0.2721 & 1.1960 & 0.25858 & 23.08 & 1.461 & 1.639 & 1.542 & 3.536 \\
Ub9 & 0.0638 & 0.2701 & 1.2844 & 0.26554 & 22.88 & 1.460 & 1.621 & 1.517 & 3.486 \\
Ub8 & 0.0642 & 0.2686 & 1.3465 & 0.27028 & 22.73 & 1.460 & 1.608 & 1.500 & 3.451 \\
Ub7 & 0.0646 & 0.2671 & 1.4055 & 0.27474 & 22.59 & 1.459 & 1.596 & 1.485 & 3.418 \\
Ub6 & 0.0651 & 0.2651 & 1.4812 & 0.28033 & 22.40 & 1.459 & 1.579 & 1.465 & 3.377 \\
Ub5 & 0.0656 & 0.2631 & 1.5534 & 0.28560 & 22.22 & 1.459 & 1.564 & 1.446 & 3.339 \\
Ub4 & 0.0659 & 0.2621 & 1.5879 & 0.28813 & 22.13 & 1.458 & 1.557 & 1.437 & 3.321 \\
Ub3 & 0.0664 & 0.2595 & 1.6730 & 0.29433 & 21.91 & 1.456 & 1.539 & 1.416 & 3.278 \\
Ub2 & 0.0669 & 0.2581 & 1.7233 & 0.29779 & 21.78 & 1.457 & 1.527 & 1.403 & 3.251 \\
Ub1 & 0.0674 & 0.2551 & 1.8120 & 0.30450 & 21.54 & 1.452 & 1.509 & 1.384 & 3.210 \\
Sb1 & 0.0682 & 0.2541 & 1.8600 & 0.30691 & 21.42 & 1.461 & 1.497 & 1.368 & 3.179 \\
Sb2 & 0.0682 & 0.2530 & 1.8845 & 0.30915 & 21.35 & 1.456 & 1.492 & 1.364 & 3.171 \\
Sb3 & 0.0684 & 0.2520 & 1.9155 & 0.31134 & 21.27 & 1.456 & 1.485 & 1.357 & 3.156 \\
Sb4 & 0.0687 & 0.2503 & 1.9620 & 0.31500 & 21.14 & 1.452 & 1.476 & 1.348 & 3.137 \\
Sb5 & 0.0703 & 0.2451 & 2.1280 & 0.32600 & 20.70 & 1.456 & 1.439 & 1.308 & 3.057 \\
Sb6 & 0.0713 & 0.2403 & 2.2610 & 0.33600 & 20.32 & 1.449 & 1.411 & 1.282 & 3.002 \\
\hline
\end{tabular}

momentum $J$, the rotational kinetic energy $T$, the gravitational binding energy $W$ and the instability parameter $\beta=T /|W|$ are defined as

$$
\begin{array}{ll}
M_{*} \equiv \int \mathrm{d}^{3} x \sqrt{\gamma} W_{L} \rho, & M \equiv \int \mathrm{d}^{3} x\left(-2 T_{0}^{0}+T_{\mu}^{\mu}\right) \alpha \sqrt{\gamma}, \\
E_{\text {int }} \equiv \int \mathrm{d}^{3} x \sqrt{\gamma} W_{L} \rho \epsilon, & J \equiv \int \mathrm{d}^{3} x T_{\phi}^{0} \alpha \sqrt{\gamma}, \\
T \equiv \frac{1}{2} \int \mathrm{d}^{3} x \Omega T_{\phi}^{0} \alpha \sqrt{\gamma}, & W \equiv T+E_{\text {int }}+M_{*}-M,
\end{array}
$$


Table 3. Same quantities as in table 1 for the sequence of models with $M_{*}=2 M_{\odot}$.

\begin{tabular}{llllllllll}
\hline Mod & $M / R_{e}$ & $\beta$ & $\rho_{c}\left(10^{-4}\right)$ & $r_{p} / r_{e}$ & $R_{e}$ & $M$ & $J / M^{2}$ & $P_{a}(\mathrm{~ms})$ & $P_{e}(\mathrm{~ms})$ \\
\hline Uc6 & 0.0841 & 0.2790 & 0.9669 & 0.21142 & 22.82 & 1.920 & 1.495 & 1.317 & 3.161 \\
Uc5 & 0.0858 & 0.2761 & 1.2663 & 0.23377 & 22.32 & 1.916 & 1.460 & 1.260 & 3.042 \\
Uc4 & 0.0880 & 0.2716 & 1.6079 & 0.25516 & 21.74 & 1.913 & 1.420 & 1.200 & 2.916 \\
Uc3 & 0.0900 & 0.2670 & 1.8982 & 0.27117 & 21.23 & 1.911 & 1.384 & 1.152 & 2.815 \\
Uc2 & 0.0917 & 0.2631 & 2.1264 & 0.28298 & 20.82 & 1.908 & 1.356 & 1.116 & 2.741 \\
Uc1 & 0.0931 & 0.2595 & 2.3176 & 0.29240 & 20.48 & 1.907 & 1.333 & 1.088 & 2.682 \\
Sc1 & 0.0970 & 0.2500 & 2.8043 & 0.31526 & 19.61 & 1.902 & 1.275 & 1.021 & 2.543 \\
Sc2 & 0.0980 & 0.2480 & 2.9091 & 0.31987 & 19.42 & 1.902 & 1.263 & 1.007 & 2.514 \\
\hline
\end{tabular}

Table 4. Same quantities as in table 1 for the sequence of models with $M_{*}=2.5 M_{\odot}$.

\begin{tabular}{llllllllll}
\hline Mod & $M / R_{e}$ & $\beta$ & $\rho_{c}\left(10^{-4}\right)$ & $r_{p} / r_{e}$ & $R_{e}$ & $M$ & $J / M^{2}$ & $P_{a}(\mathrm{~ms})$ & $P_{e}(\mathrm{~ms})$ \\
\hline Ud15 & 0.1113 & 0.2771 & 1.3117 & 0.21259 & 21.29 & 2.369 & 1.320 & 1.025 & 2.630 \\
Ud14 & 0.1124 & 0.2760 & 1.4608 & 0.22121 & 21.07 & 2.368 & 1.307 & 1.005 & 2.588 \\
Ud13 & 0.1132 & 0.2750 & 1.5826 & 0.22785 & 20.90 & 2.366 & 1.298 & 0.990 & 2.555 \\
Ud12 & 0.1140 & 0.2740 & 1.6961 & 0.23372 & 20.74 & 2.364 & 1.289 & 0.976 & 2.526 \\
Ud11 & 0.1149 & 0.2730 & 1.8103 & 0.23925 & 20.58 & 2.364 & 1.280 & 0.962 & 2.496 \\
Ud10 & 0.1156 & 0.2721 & 1.9104 & 0.24399 & 20.44 & 2.362 & 1.272 & 0.950 & 2.471 \\
Ud9 & 0.1164 & 0.2710 & 2.0153 & 0.24868 & 20.29 & 2.362 & 1.264 & 0.938 & 2.445 \\
Ud8 & 0.1171 & 0.2701 & 2.1102 & 0.25286 & 20.16 & 2.360 & 1.256 & 0.927 & 2.423 \\
Ud7 & 0.1186 & 0.2680 & 2.3022 & 0.26083 & 19.89 & 2.359 & 1.241 & 0.906 & 2.377 \\
Ud6 & 0.1189 & 0.2675 & 2.3534 & 0.26291 & 19.82 & 2.358 & 1.238 & 0.901 & 2.366 \\
Ud5 & 0.1196 & 0.2666 & 2.4336 & 0.26609 & 19.71 & 2.357 & 1.231 & 0.892 & 2.348 \\
Ud4 & 0.1207 & 0.2650 & 2.5698 & 0.27128 & 19.52 & 2.356 & 1.221 & 0.878 & 2.318 \\
Ud3 & 0.1220 & 0.2631 & 2.7402 & 0.27760 & 19.29 & 2.353 & 1.208 & 0.861 & 2.282 \\
Ud2 & 0.1254 & 0.2580 & 3.1583 & 0.29210 & 18.74 & 2.349 & 1.178 & 0.821 & 2.198 \\
Ud1 & 0.1302 & 0.2510 & 3.7335 & 0.31027 & 18.00 & 2.343 & 1.138 & 0.772 & 2.094 \\
Sd1 & 0.1314 & 0.2491 & 3.8899 & 0.31503 & 17.81 & 2.341 & 1.127 & 0.760 & 2.068 \\
Sd2 & 0.1321 & 0.2480 & 3.9735 & 0.31745 & 17.71 & 2.340 & 1.122 & 0.753 & 2.054 \\
Sd3 & 0.1329 & 0.2470 & 4.0607 & 0.31991 & 17.61 & 2.340 & 1.116 & 0.746 & 2.040 \\
Sd4 & 0.1341 & 0.2450 & 4.2188 & 0.32450 & 17.42 & 2.337 & 1.106 & 0.735 & 2.016 \\
Sd5 & 0.1376 & 0.2400 & 4.6380 & 0.33596 & 16.95 & 2.333 & 1.081 & 0.706 & 1.955 \\
Sd6 & 0.1412 & 0.2350 & 5.0710 & 0.34714 & 16.49 & 2.329 & 1.056 & 0.678 & 1.897 \\
\hline
\end{tabular}

where $\alpha$ is the lapse function, $\sqrt{\gamma}$ is the square root of the three-dimensional metric determinant and $W_{L}=\alpha u^{0}$ is the fluid Lorentz factor. We stress that the definitions (5)-(7) of quantities such as $J, T, W$ and $\beta$ are meaningful only in the case of stationary axisymmetric configurations and should therefore be treated with care once the rotational symmetry is lost.

Traditionally, numerical simulations of the dynamical bar-mode instability have sometimes been sped up by introducing very large $m=2$ deformations in the initial condition. As discussed in paper I, the introduction of any perturbation (especially when this is not a small one) may lead to spurious effects and erroneous interpretations. Being aware of this, we used, only in some selected simulations below the threshold, initial density perturbations of the type

$$
\delta \rho_{2}(x, y, z)=\delta_{2}\left(\frac{x^{2}-y^{2}}{r_{e}^{2}}\right) \rho,
$$


where $\delta_{2}$ is the amplitude of the $m=2$ perturbation (which we set to be $\delta_{2} \simeq 0.01-0.04$ ). This perturbation has then the effect of superimposing on the axially symmetric initial model a bar deformation that is larger than the (unavoidable) $m=4$-mode perturbation introduced by the Cartesian grid discretization. The introduction of such perturbation allowed us to estimate the frequency of the $m=2$ mode below the threshold for the onset of the instability and reduce considerably the computing costs in a region of the parameter space where the instability does not develop.

We solve the Einstein equations (1) formulated as a first-order (in time) quasi-linear [8] system of equations, where the independent variables are the three-metric $\gamma_{i j}$ and the extrinsic curvature $K_{i j}$. In particular, we use the conformal traceless reformulation of the ADM system of evolution equations, first suggested in [9], in which the evolved variables are the conformal factor $\phi$, the trace of the extrinsic curvature $K$, the conformal 3-metric $\tilde{\gamma}_{i j}$, the conformal traceless extrinsic curvature $\tilde{A}_{i j}$ and the conformal connection functions $\tilde{\Gamma}^{i}$. The solution of the hydrodynamics equations is obtained by using the general-relativistic hydrodynamics code Whisky, in which the hydrodynamics equations are written as finite differences on a Cartesian grid and solved using high-resolution shock-capturing schemes, as described in [10]. During the evolution we use the 'ideal-fluid' EOS: $p=(\Gamma-1) \rho \epsilon$. Full details of the numerical scheme and the gauge conditions used are reported in paper I.

\section{Methodology of the analysis}

A number of different quantities are calculated during the evolution to monitor the dynamics of the instability. Among them is the quadrupole moment of the matter distribution, which we compute in terms of the conserved density $\sqrt{\gamma} W_{L} \rho$ rather than of the rest-mass density $\rho$ or of the $T_{00}$ component of the stress energy momentum tensor

$$
I^{j k}=\int \mathrm{d}^{3} x \sqrt{\gamma} W_{L} \rho x^{j} x^{k} .
$$

Of course, the use of $\sqrt{\gamma} W_{L} \rho$ in place of $\rho$ or of $T_{00}$ is arbitrary and all three expressions would have the same Newtonian limit. However, we prefer the form (9) because $\sqrt{\gamma} W_{L} \rho$ is a quantity whose conservation is guaranteed by the form chosen for the hydrodynamics equations. The quantity (9) can be conveniently used to quantify both the growth time of the instability $\tau_{2}$ and the oscillation frequency of the unstable bar-mode once the instability is fully developed $f_{2}$. (Hereafter we will indicate respectively with $\tau_{i}$ and $f_{i}$ the growth time and frequency of the $m=i$ unstable mode.)

In practice, we perform a nonlinear least-squares fit of the computed quadrupole $I^{j k}(t)$ and we generally use the $x y$ component, with the trial functions

$$
I^{j k}(t)=\left(I^{j k}\right)_{0} \mathrm{e}^{t / \tau_{2}} \cos \left(2 \pi f_{2} t+\phi_{0}\right) .
$$

Furthermore, we define the modulus $I(t)$ of the two components of the quadrupole in the $x y$ plane and the distortion parameter $\eta(t)$ as

$$
I \equiv \frac{1}{2} \sqrt{\left(2 I^{x y}\right)^{2}+\left(I^{x x}-I^{y y}\right)^{2}}, \quad \eta \equiv \frac{I}{2\left(I^{x x}+I^{y y}\right)},
$$

and the instantaneous orientation of the bar is given by

$$
\phi_{\mathrm{bar}}=\tan ^{-1}\left(\frac{2 I^{x y}}{I^{x x}-I^{y y}}\right) .
$$

Finally, as a useful tool to describe the nonlinear properties of the development and saturation of the instability, the rest-mass density is decomposed into its Fourier modes $P_{m}(t)$ :

$$
P_{m} \equiv \int \mathrm{d}^{3} x \rho \mathrm{e}^{\mathrm{i} m \phi}
$$


The phase $\phi_{m} \equiv \arg \left(P_{m}\right)$ essentially provides the instantaneous orientation of the $m$ th mode when the corresponding mode has a nonzero power. Note that despite their denomination, the Fourier modes (13) do not represent proper eigenmodes of oscillation of the star. While, in fact, the latter are well defined only within a perturbative regime, the former simply represent a tool to quantify, within the fully nonlinear regime, what are the main components of the rest-mass distribution. Stated differently, we do not expect quasi-normal modes of oscillations to be present except in the initial and final stages of the instability, for which a perturbative description is adequate.

While all quantities (9)-(13) are expressed in terms of the coordinate time $t$ and do not represent therefore invariant measurements, the lengthscale of variation of the lapse function at any given time is always larger than twice the stellar radius at that time, ensuring that events on the same timeslice are also close in proper time. As representative examples, we note that for the most compact model the values of the lapse at the centre of the star, at its surface and at the outer boundary are $0.67,0.84$ and 0.95 , respectively. Similarly, the corresponding values for the least-compact model are 0.92, 0.95 and 0.98 , respectively.

The simulations have been carried out on a grid with a uniform resolution of $\Delta x / M_{\odot}=0.625$ and outer boundaries at $48.75 M_{\odot}$, where 'radiative' boundary conditions (i.e., Sommerfeld outgoing boundary conditions) are applied to the field variables, while the fluid variables are simply not evolved. Such outer boundaries are sufficiently far from the surface of the star to make the use of mesh refinements in Whisky not necessary (see also section VI E of paper I for a more detailed discussion of the role of the grid size on the evolution of the instability). Furthermore, for those models used in the extrapolation technique and that are largely over-critical (see section 4.1), we have imposed a 'bitant' symmetry (i.e., $z \rightarrow-z$ ) and a ' $\pi$-symmetry' (i.e., a $180^{\circ}$ rotation around the $z$-axis) in order to reduce the size of the computational domain by a factor of 4 .

\section{Effects of the compactness}

\subsection{Threshold of the $m=2$ instability}

The determination of the dependence on $\beta$ of the frequencies and of the growth times of the $m=2$ bar-mode instability in the region near the threshold is particularly delicate as the models are only slightly over-critical, with very small growth rates and hence the simulations are computationally very expensive. For this purpose we here use an extrapolation technique already described in paper I, where it was shown to be both accurate and robust. In essence, we exploit the results of the classical Newtonian study of the bar-mode instability of Maclaurin spheroids of incompressible and self-gravitating Newtonian fluid in equilibrium [11], extrapolating, via suitable fits, its predictions to a general-relativistic context. We recall, in fact, that in the classical scenario the eigenfrequency of the $m=2$ bar-mode can be expressed in terms of two real and differentiable functions of $\beta, \Omega \equiv 2 \pi f_{2}$ and $1 / \tau_{2}^{2}$, in a relation of the type

$$
\sigma=\Omega(\beta) \pm \frac{\mathrm{i}}{\sqrt{\tau_{2}^{2}(\beta)}} .
$$

The bar-mode becomes unstable when the function $1 / \tau_{2}^{2}$ changes sign, with the square root going from being imaginary to being real. The value of $\beta$ at which this change of sign happens represents then the threshold for the onset of the instability $\beta_{c}$; clearly, for models above the threshold, $\Omega / 4 \pi$ and $\tau_{2}$ represent the pattern speed and the growth time of the unstable bar deformation of the considered star model, respectively. 
Table 5. Least-squares fit of the value of $\beta$ at the threshold for the development of the bar-mode instability for the four series of models reported in table 8 . The critical value for the onset of the instability $\beta_{c}$ is the value of $\beta$ for $1 / \tau_{2}^{2}=0$ ( $\tau$ is measured in ms) and the digits in brackets represent the error in the fit. The results of the fits are shown in figure 3.

\begin{tabular}{llll}
\hline$M_{*}=1.0 M_{\odot}$ & $\beta=0.2598(8)$ & $+0.0379(19)$ & $\left(1 / \tau_{2}\right)^{2}$ \\
$M_{*}=1.5 M_{\odot}$ & $\beta=0.2558(5)$ & $+0.0236(8)$ & $\left(1 / \tau_{2}\right)^{2}$ \\
$M_{*}=2.0 M_{\odot}$ & $\beta=0.2528(15)$ & $+0.0161(13)$ & $\left(1 / \tau_{2}\right)^{2}$ \\
$M_{*}=2.5 M_{\odot}$ & $\beta=0.2494(14)$ & $+0.0116(8)$ & $\left(1 / \tau_{2}\right)^{2}$ \\
\hline
\end{tabular}

Table 6. Least-squares fit of the value of the frequency $f_{\mathrm{B}}$ (in $\mathrm{Hz}$ ) of the bar-mode $(10)$ at the threshold for the onset of the bar-mode instability as a function of $\theta \equiv\left(\beta-\beta_{c}\right) / \beta_{c}$ for the four series of models at constant baryonic mass. The value of the frequency $f_{2}$ at the threshold is the value for $\theta=0$ and the digits in brackets represent the error in the fit.

\begin{tabular}{llll}
\hline$M_{*}=1.0 M_{\odot}$ & $f_{\mathrm{B}}=384(7)$ & $-8(25) 10 \theta$ & $-6(2) 1000 \theta^{2}$ \\
$M_{*}=1.5 M_{\odot}$ & $f_{\mathrm{B}}=551(8)$ & $-4(3) 100 \theta$ & $-6(2) 1000 \theta^{2}$ \\
$M_{*}=2.0 M_{\odot}$ & $f_{\mathrm{B}}=738(32)$ & $-7(11) 100 \theta$ & $-6(8) 1000 \theta^{2}$ \\
$M_{*}=2.5 M_{\odot}$ & $f_{\mathrm{B}}=991(10)$ & $-10(3) 100 \theta$ & $-9(2) 1000 \theta^{2}$ \\
\hline
\end{tabular}

With the rather reasonable assumption that the two functions $\Omega$ and $1 / \tau_{2}^{2}$ are regular also in full general relativity, we expand them in a Taylor series around the threshold and express them in terms of five unknown coefficients $f_{c}, f_{c}^{(1)}, f_{c}^{(2)}, k, \beta_{c}$, i.e.

$$
\begin{aligned}
& \frac{\Omega(\beta)}{2 \pi} \approx f_{c}+f_{c}^{(1)} \frac{\left(\beta-\beta_{c}\right)}{\beta_{c}}+f_{c}^{(2)} \frac{\left(\beta-\beta_{c}\right)^{2}}{\beta_{c}^{2}}+\mathcal{O}\left(\left(\beta-\beta_{c}\right)^{3}\right), \\
& \frac{1}{\tau_{2}^{2}} \approx \frac{1}{k^{2}}\left(\beta-\beta_{c}\right)+\mathcal{O}\left(\left(\beta-\beta_{c}\right)^{2}\right) .
\end{aligned}
$$

Expressions (15) and (16) represent very good approximations to the actual data and the five parameters can be determined straightforwardly by fitting the pattern speeds and the growth times obtained in the largely over-critical models. For these models, we recall, the development of the $m=2$ bar-mode deformation is very rapid, the extraction of the instability parameter is robust and it can be safely simulated even at rather low resolutions (see paper I).

In practice, using the data obtained from the simulations of the four sequences of initial models with constant baryonic mass, we have computed the values for $f_{2}$ and $\tau_{2}$ by means of a nonlinear least-squares fit to the trial form of equation (10). Making use of these results, which are collected in table 8 , we have then computed the unknown coefficients $f_{c}, f_{c}^{(1)}, f_{c}^{(2)}, k$ and $\beta_{c}$ through a least-squares fit of the values for $f_{2}$ and $\tau_{2}$ once expressed as functions of $\beta$ as in equations (15) and (16).

The results of these fits are reported in tables 5 and 6 and summarized in figure 2 . In particular, for each of the four sequences this figure shows with solid lines the two fitted curves for $\Omega(\beta)$ and $\tau_{2}(\beta)$, and with dotted lines the corresponding extrapolations for models below the threshold. In addition, different symbols are used to mark the results of the numerical simulations, with 'bare' error bars denoting the unperturbed unstable models (as reported in table 8 and which have been used for the fits), triangles denoting the unperturbed models (as reported in table 8 and not used for the fits because of the large error in determining their evolution parameters), squares denoting the stable perturbed models (as reported in table 7) and open circles denoting the models dominated by the $m=3$ instability (and again reported in table 7). It is worth noting that for these last models the frequency of the $m=2$ mode is considerably altered by the growing $m=3$ deformation, which rapidly dominates the small initial $m=2$ bar-mode perturbation used for the simulation of these models. 


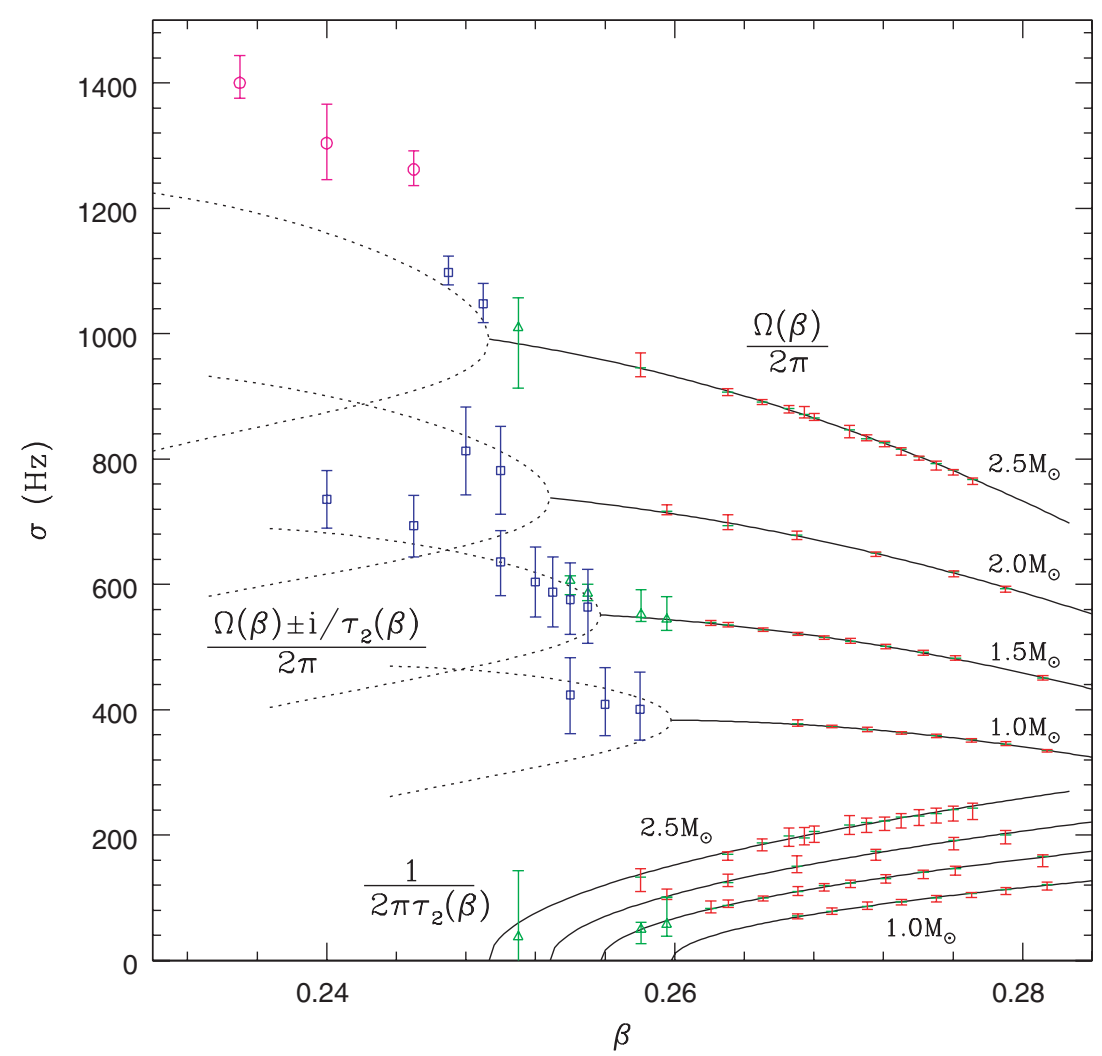

Figure 2. Critical diagram as constructed with the frequencies and growth times relative to the unperturbed models of table 8 . The solid lines represent the two fitted curves for $\Omega(\beta)$ and $\tau_{2}(\beta)$, while the dotted lines the corresponding extrapolations below the threshold. Triangles refer to the unperturbed models of table 8 that have not been used for the fit, squares to the perturbed models of table 7 (squares), and open circles to the models dominated by the $m=3$ deformation.

We also note that the error bars in table 8 are computed in different ways for the growth rates and for the frequencies. In the first case they are computed as the difference between the minimum and maximum values of $\mathrm{d} \log (I(t)) / \mathrm{d} t$ in the time intervals in which the quadrupolar deformation lies between the $5 \%$ and the $35 \%$ of its maximum amplitude. In the second case, instead, the error bars are determined using the minimum and maximum values, in the same time interval, of the pattern speeds extracted from the collective phase $\phi_{\text {bar }}(t)$ (cf equation (12)). The frequencies and the error bars for the stable models reported in table 7 and figure 2, on the other hand, are computed using Lomb's power spectrum analysis [12], which is better suited to study a signal spanning over a short time interval and which is comparable with the main frequency of the Fourier transform. In particular, they refer to the frequency at which the Fourier transform of the imaginary part of $P_{m}(t)$ has its maximum, while the error bars to the interval in frequencies where the Fourier transform is above $1 / 2$ of its maximum value.

The left panel of figure 3 offers a different view of the data already reported in figure 2 by showing $\beta$ as a function of $1 / \tau^{2}$ for the four sequences considered and thus highlighting the very good approximation in the ansatz (16). Indicated with open circles are also the extrapolations of the critical value $\beta_{c}$ and these are then reported in the right panel of figure 3 as a function of the baryonic mass $M_{*}$ of the four sequences. Clearly, they show a 
Table 7. For models below the threshold for the onset of the $m=2$ bar-mode instability the table reports the measured frequencies $f_{2}$ of the bar deformation as well as the frequencies $f_{3}$ and growth times $\tau_{3}$ of the $m=3$ deformation. For each model we also indicate the value of $\beta$, the grid resolution $\Delta x / M_{\odot}$ and the initial bar-mode perturbation $\delta_{2}$. All models have been evolved without a $\pi$-symmetry.

\begin{tabular}{|c|c|c|c|c|c|c|}
\hline Model & $\beta$ & $\Delta x / M_{\odot}$ & $\delta_{2}$ & $f_{2}(\mathrm{~Hz})$ & $f_{3}(\mathrm{~Hz})$ & $\tau_{3}(\mathrm{~ms})$ \\
\hline $\mathrm{Sa} 3$ & 0.254 & 0.5 & 0.30 & $424_{-62}^{+59}$ & * & * \\
\hline $\mathrm{Sa} 2$ & 0.256 & 0.5 & 0.01 & $409_{-50}^{+58}$ & * & * \\
\hline Sa1 & 0.258 & 0.5 & 0.01 & $401_{-49}^{+59}$ & * & * \\
\hline Sb6 & 0.240 & 0.5 & 0.04 & $736_{-46}^{+46}$ & $*$ & $*$ \\
\hline Sb5 & 0.245 & 0.5 & 0.04 & $694_{-50}^{+48}$ & * & * \\
\hline $\mathrm{Sb} 4$ & 0.250 & 0.5 & 0.04 & $636_{-54}^{+50}$ & * & * \\
\hline $\mathrm{Sb} 3$ & 0.252 & 0.5 & 0.04 & $604_{-56}^{+56}$ & * & * \\
\hline $\mathrm{Sb} 2$ & 0.253 & 0.5 & 0.04 & $588_{-56}^{+56}$ & * & * \\
\hline Sb1 & 0.254 & 0.5 & 0.04 & $576_{-56}^{+58}$ & * & * \\
\hline Ub1 & 0.255 & 0.5 & 0.04 & $564_{-58}^{+60}$ & * & * \\
\hline Sb1 & 0.254 & 0.5 & 0.0 & $606_{-21}^{+8}$ & $1302_{+12}^{-12}$ & 3.8 \\
\hline Ub1 & 0.255 & 0.5 & 0.0 & $586_{-12}^{+14}$ & $1284_{+16}^{-12}$ & 10.0 \\
\hline Sc1 & 0.250 & 0.625 & 0.01 & $782_{-70}^{+70}$ & * & * \\
\hline $\mathrm{Sc} 2$ & 0.248 & 0.625 & 0.01 & $818_{-70}^{+70}$ & $*$ & * \\
\hline $\mathrm{Sc} 2$ & 0.248 & 0.625 & 0.0 & $\star$ & $1746_{-14}^{+12}$ & 4.0 \\
\hline Sd6 & 0.235 & 0.625 & 0.01 & $1400_{-24}^{+44}$ & $2454_{-58}^{+52}$ & 2.7 \\
\hline $\mathrm{Sd} 5$ & 0.240 & 0.625 & 0.01 & $1304_{-58}^{+62}$ & $2312_{-100}^{+122}$ & 1.4 \\
\hline $\mathrm{Sd} 4$ & 0.245 & 0.625 & 0.01 & $1262_{-26}^{+30}$ & $2248_{-76}^{+66}$ & 2.9 \\
\hline $\mathrm{Sd} 3$ & 0.247 & 0.625 & 0.01 & $1098_{-20}^{+26}$ & $2200_{-54}^{+62}$ & 2.5 \\
\hline $\mathrm{Sd} 1$ & 0.249 & 0.625 & 0.01 & $1048_{-30}^{+32}$ & $2136_{-50}^{+74}$ & 3.5 \\
\hline Sd6 & 0.235 & 0.625 & 0.0 & $\star$ & $2474_{-20}^{+18}$ & 2.7 \\
\hline Sd5 & 0.240 & 0.625 & 0.0 & $\star$ & $2372_{-18}^{+20}$ & 2.7 \\
\hline $\mathrm{Sd} 4$ & 0.245 & 0.625 & 0.0 & $\star$ & $2296_{-16}^{+16}$ & 2.2 \\
\hline $\mathrm{Sd} 2$ & 0.248 & 0.625 & 0.0 & $\star$ & $2228_{-20}^{+22}$ & 2.5 \\
\hline Sd1 & 0.249 & 0.625 & 0.0 & $1446_{-26}^{+32}$ & $2174_{-28}^{+28}$ & 2.2 \\
\hline Ud1 & 0.251 & 0.625 & 0.0 & $1022_{-56}^{+32}$ & $2158_{-16}^{+12}$ & 2.9 \\
\hline
\end{tabular}

* A dynamical $m=3$ deformation instability cannot be detected.

$\star$ The frequency of the $m=2$ bar-mode cannot be measured.

linear dependence on $M_{*}$, which can be expressed phenomenologically as

$$
\beta_{c}\left(M_{*}\right)=0.266(1)-0.0070(3)\left(\frac{M_{*}}{M_{\odot}}\right),
$$

and allows us to extrapolate the value of the threshold in the limit of a zero baryonic mass. Interestingly, the resulting number $\beta_{c}\left(M_{*}=0\right)=0.266$ is in very good agreement with the value of $\beta_{c}=0.266$ obtained in Newtonian gravity [13] through a linear stability analysis for a sequence of equilibrium models with the same polytropic index and degree of differential rotation as used here. This agreement represents an additional confirmation of the accuracy and robustness of our extrapolation method in determining the position of the threshold. 
Table 8. For models above the threshold the table reports the measured frequencies $f_{2}$ and growth times $\tau_{2}$ of the $m=2$ deformation as well as the value of $\beta$. All models have been evolved with a grid resolution $\Delta x / M_{\odot}=0.625$ and no initial perturbation; furthermore, a $\pi$-symmetry has been used for all models with the exception of models Ub1 and Ud1 that are closer to the threshold.

\begin{tabular}{|c|c|c|c|c|c|c|c|}
\hline Model & $\beta$ & $\tau_{2}(\mathrm{~ms})$ & $f_{2}(\mathrm{~Hz})$ & Model & $\beta$ & $\tau_{2}(\mathrm{~ms})$ & $f_{2}(\mathrm{~Hz})$ \\
\hline Ua1 & 0.2671 & $2.235_{-0.09}^{+0.16}$ & $377_{-4}^{+7}$ & Uc1 & 0.2596 & $1.564_{-0.17}^{+0.07}$ & $717_{-6}^{+10}$ \\
\hline Ua2 & 0.2690 & $2.019_{-0.13}^{+0.14}$ & $373_{-1}^{+3}$ & Uc2 & 0.2631 & $1.277_{-0.12}^{+0.07}$ & $694_{-6}^{+18}$ \\
\hline Ua3 & 0.2711 & $1.838_{-0.14}^{+0.11}$ & $368_{-3}^{+4}$ & Uc3 & 0.2670 & $1.055_{-0.10}^{+0.08}$ & $679_{-7}^{+7}$ \\
\hline Ua4 & 0.2730 & $1.702_{-0.06}^{+0.10}$ & $364_{-3}^{+1}$ & Uc4 & 0.2716 & $0.915_{-0.02}^{+0.08}$ & $650_{-5}^{+2}$ \\
\hline Ua5 & 0.2750 & $1.600_{-0.06}^{+0.10}$ & $359_{-4}^{+2}$ & Uc5 & 0.2761 & $0.829_{-0.02}^{+0.07}$ & $621_{-8}^{+1}$ \\
\hline Ua6 & 0.2771 & $1.482_{-0.02}^{+0.11}$ & $352_{-4}^{+1}$ & Uc6 & 0.2790 & $0.795_{-0.03}^{+0.06}$ & $593_{-6}^{+4}$ \\
\hline Ua7 & 0.2790 & $1.405_{-0.04}^{+0.11}$ & $345_{-2}^{+4}$ & Ud1 & 0.2510 & $\approx 4.2$ & $1009_{-96}^{+48}$ \\
\hline $\mathrm{Ua} 8$ & 0.2814 & $1.313_{-0.04}^{+0.10}$ & $335_{-2}^{+2}$ & $\mathrm{Ud} 2$ & 0.2580 & $1.193_{-0.11}^{+0.25}$ & $945_{-14}^{+24}$ \\
\hline Ub1 & 0.2551 & $*$ & $586_{-12}^{+14}$ & Ud3 & 0.2631 & $0.938_{-0.02}^{+0.05}$ & $907_{-6}^{+6}$ \\
\hline Ub2 & 0.2581 & $3.215_{-0.61}^{+2.62}$ & $552_{-11}^{+40}$ & Ud4 & 0.2650 & $0.846_{-0.03}^{+0.06}$ & $891_{-4}^{+4}$ \\
\hline Ub3 & 0.2595 & $2.758_{-1.17}^{+1.32}$ & $544_{-17}^{+37}$ & Ud5 & 0.2666 & $0.800_{-0.05}^{+0.07}$ & $881_{-7}^{+5}$ \\
\hline Ub4 & 0.2621 & $1.920_{-0.25}^{+0.17}$ & $540_{-5}^{+3}$ & Ud6 & 0.2675 & $0.813_{-0.06}^{+0.05}$ & $871_{-6}^{+13}$ \\
\hline Ub5 & 0.2631 & $1.803_{-0.15}^{+0.07}$ & $535_{-3}^{+4}$ & Ud7 & 0.2680 & $0.774_{-0.03}^{+0.07}$ & $865_{-4}^{+7}$ \\
\hline Ub6 & 0.2651 & $1.591_{-0.04}^{+0.08}$ & $527_{-2}^{+4}$ & Ud8 & 0.2701 & $0.735_{-0.05}^{+0.05}$ & $847_{-13}^{+7}$ \\
\hline Ub7 & 0.2671 & $1.441_{-0.09}^{+0.10}$ & $520_{-1}^{+4}$ & Ud9 & 0.2710 & $0.724_{-0.02}^{+0.05}$ & $832_{-3}^{+6}$ \\
\hline Ub8 & 0.2686 & $1.335_{-0.03}^{+0.10}$ & $516_{-3}^{+2}$ & Ud10 & 0.2721 & $0.716_{-0.02}^{+0.05}$ & $826_{-7}^{+2}$ \\
\hline Ub9 & 0.2701 & $1.290_{-0.05}^{+0.07}$ & $509_{-3}^{+5}$ & Ud11 & 0.2730 & $0.697_{-0.02}^{+0.06}$ & $816_{-9}^{+3}$ \\
\hline Ub10 & 0.2721 & $1.214_{-0.05}^{+0.08}$ & $502_{-4}^{+3}$ & Ud12 & 0.2741 & $0.690_{-0.03}^{+0.05}$ & $804_{-6}^{+1}$ \\
\hline Ub11 & 0.2743 & $1.126_{-0.02}^{+0.09}$ & $492_{-5}^{+2}$ & Ud13 & 0.2750 & $0.679_{-0.02}^{+0.05}$ & $793_{-11}^{+4}$ \\
\hline Ub12 & 0.2761 & $1.087_{-0.03}^{+0.08}$ & $483_{-3}^{+4}$ & Ud14 & 0.2760 & $0.660_{-0.01}^{+0.06}$ & $782_{-8}^{+1}$ \\
\hline Ub13 & 0.2812 & $0.959_{-0.01}^{+0.10}$ & $450_{-3}^{+4}$ & Ud15 & 0.2771 & $0.654_{-0.02}^{+0.05}$ & $767_{-8}^{+2}$ \\
\hline
\end{tabular}

$\star$ The growth rate of the $m=2$ bar-mode cannot be reliably measured for this model.

Using the phenomenological dependence of the threshold $\beta_{c}$ on the stellar rest mass given by expression (17), we have also reconstructed the dependence of $\beta_{c}$ on the stellar compactness $M / R_{e}$. In practice, for a large number of values of $M_{*}$ between 0 and $2.5 M_{\odot}$ we have computed the value of the proper equatorial radius $R_{e}$ and of the gravitational mass $M$ of the corresponding stellar model in equilibrium that lies on the line defined by equation (17). The result of this is shown as a solid line in figure 1, with the dashed band representing the estimated error obtained using the least-squares fitting. Furthermore, a good polynomial reconstruction of the median of the error bar in figure 1 suggests a quadratic dependence of the threshold on the compactness, with coefficients given by

$$
\beta_{c}=0.266-0.18\left(\frac{M}{R_{e}}\right)+0.36\left(\frac{M}{R_{e}}\right)^{2} \text {. }
$$

Once perturbative calculations are developed in the regime of rapid and differential rotation considered here, expression (18) can be used as a guideline to the perturbative approach and the numerical measurements of the threshold can be used to assess the validity and accuracy of the perturbative approximation.

Overall, these results represent the first quantitative determination of the dependence of the critical $\beta$ on the compactness of the star for a selected profile of differential rotation and EOS. A similar investigation was carried out also in [14] using the post-Newtonian (PN) 

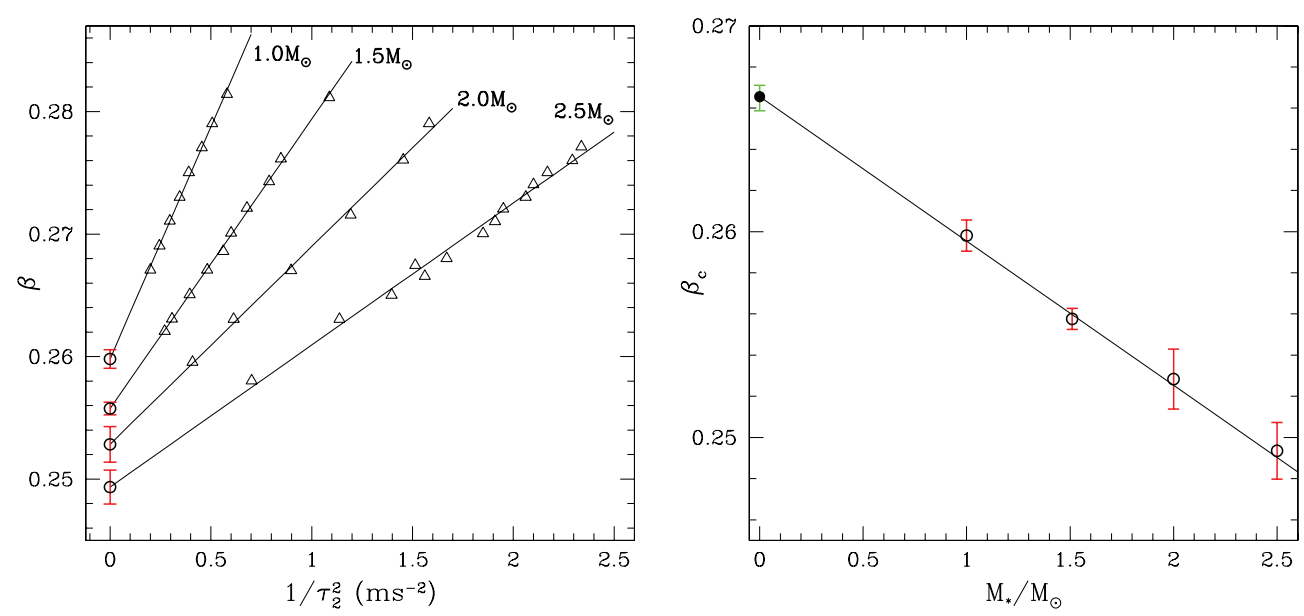

Figure 3. Left panel: indicated with triangles are the data already reported in figure 2 but shown here as a function of $1 / \tau^{2}$ for the four constant-rest-mass sequences considered. The open circles indicate instead the extrapolation to $\beta_{c}$. The numerical values of the four fits are reported in table 5 . Right panel: the open circles are the same as in the left panel but shown as a function of the baryonic mass $M_{*}$; the filled circle, on the other hand, represents the limit of zero rest-mass and can be compared with the threshold value calculated in Newtonian gravity and with a linear stability analysis in [13].

approximation and rather large initial $m=2$ perturbations with $\delta_{2}=0.1$. While the results of that analysis had the limitation of the PN approximation and were affected by the large initial perturbations, they also provided the first evidence that the threshold is smaller for stellar models with larger compactness.

\subsection{Persistence of the bar}

As mentioned in the introduction and for all of the models considered here, any nonaxisymmetric deformation that develops as a result of a dynamical instability is also suppressed over a time comparable (i.e., of the order of a few) dynamical timescales. In addition, we have found that the persistence of the bar deformation increases as $\beta-\beta_{c}$ tends to zero. Besides confirming what is already discussed in paper I, this behaviour matches the expectation that the persistence of the bar is related to the degree of overcriticality, with the duration of the saturation increasing as the threshold is approached. For a star with a small compactness this time would tend to the radiation-reaction timescale for a model with $\beta=\beta_{c}$ and to zero for a model with $\beta \gg \beta_{c}$. For a star with large compactness, on the other hand, the persistence near the threshold can be further reduced by the stronger gravitational fields.

Here, we also intend to gain insight on the role played by the stellar compactness on the persistence of the bar deformations and, to this scope, we have extended the time over which the simulations are carried out for some selected models. Figure 4 summarizes the results of these extended simulations by reporting the evolution of the distortion parameter $\eta$ for two models, i.e., Ub4 (dotted line) and Ud2 (solid line), having different masses and compactnesses (i.e., $M=1.5 M_{\odot}, M=2.5 M_{\odot}$ and $M / R_{e} \simeq 0.066, M / R_{e} \simeq 0.125$, respectively), but with a similar distance from the threshold (i.e., $\beta-\beta_{c} \simeq 0.007-0.008$ ). The two evolutions have been suitably shifted in time so as to have the maximum deformation at $t=0$.

Both of these models have been evolved using a $\pi$-symmetry in order to remove the effect of the odd-mode coupling on the suppression of the bar deformation and yet they 


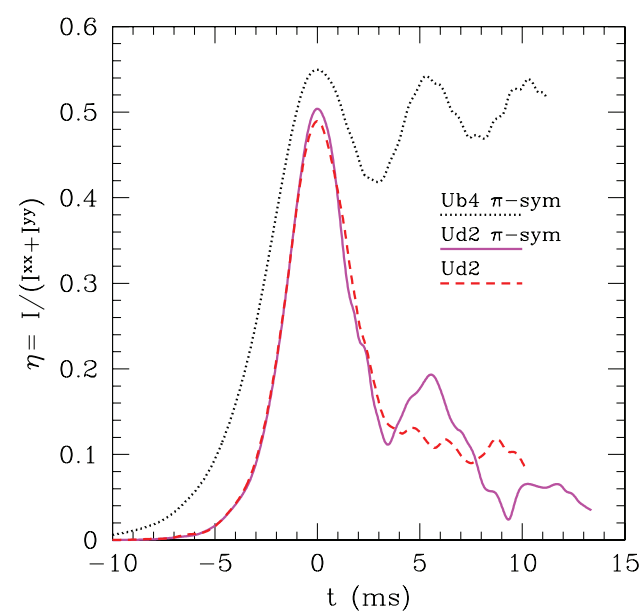

Figure 4. Comparison of the evolution of the distortion parameters $\eta$ for a low-mass, lowcompactness model Ub4 and a high-mass, high-compactness model Ud2. Indicated with a dotted and a solid line are the simulations for Ub4 and Ud2 using $\pi$-symmetry, while indicated with a dashed line is the evolution for model Ud2 without $\pi$-symmetry. All the evolutions have been suitably shifted in time so as to have the maximum deformation at $t=0$.

show a remarkable difference. The low-mass, low-compactness model Ub4 reaches and maintains a rather large bar deformation over several milliseconds of evolution. The high-mass, high-compactness model Ud2, on the other hand, reaches comparable deformations but these are rapidly suppressed over a few milliseconds, despite the use of the $\pi$-symmetry. This behaviour underlines something that was already remarked in paper I, namely that, depending on the specific stellar properties, the bar deformation can be suppressed also by factors other than the mode coupling, which is most effective for models near the threshold. In particular, for low-mass models (such as model U13 in paper I) this effect is simply the excess of rotational kinetic energy which is efficiently converted into internal energy (see discussion in section VIB of paper I). For high-mass and high-compactness models (such as model Ud2 above), on the other hand, the strength of the gravitational field, together with the excess kinetic energy, are very efficient in suppressing the bar deformation. This was not evident in the stellar models considered in paper I, which all had $M_{*}=1.5 M_{\odot}$.

To confirm that the $m=1$-mode coupling plays no significant role in the dynamics of model Ud2, we show with a dashed line in figure 4 the evolution of the bar deformation for model Ud2 in a simulation in which the $\pi$-symmetry was not enforced. As is obvious in the comparison between the solid and dashed lines, the lack of symmetry does not change the suppression of the bar-mode deformation and a difference emerges only at $t \sim 5 \mathrm{~ms}$, when the simulation with $\pi$-symmetry tends to revive the bar deformation, while this does not happen in the simulation without the $\pi$-symmetry.

\subsection{Unstable deformations with $m=3$}

While studying the dynamics of models with $M_{*} \geqslant 2 M_{\odot}$ and values of $\beta$ near the threshold for the development of the bar-mode instability, we have also found stellar models that show the development of a dynamical instability with a dominant $m=3$ deformation. Interestingly, this instability developed without the introduction of any $m=3$ initial perturbation as was instead done in [15]. More precisely, we found four models (Sc2, Sd6, Sd5, Sd4) for which 

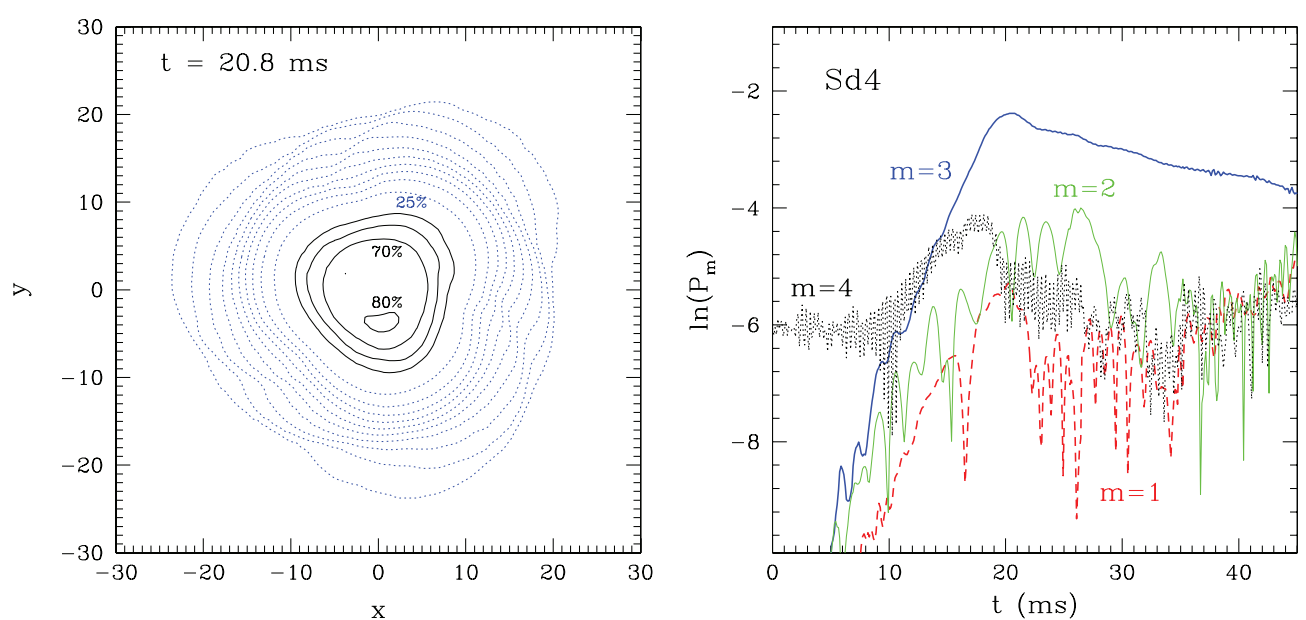

Figure 5. Left panel: rest-mass isodensity for model Sd4. Starting from the centre the solid lines represent respectively: $80 \%, 70 \%, 60 \%$ and $50 \%$ of the maximum density. Dotted lines are $1 / 2^{n}$ times the maximum density, with $n=2, \ldots, 11$. The snapshot was taken at $t=20.8 \mathrm{~ms}$. Right panel: evolution of the global rest-mass density modes defined in equation (13) for the same simulation.

the $m=3$ mode is the fastest-growing dynamically unstable deformation. In addition, we also see evidence of a dynamical unstable growth of the $m=3$ mode for models Sb1, Ub1, $\mathrm{Sd} 3, \mathrm{Sd} 2, \mathrm{Sd} 1$ and Ud1. However, because all the above models lay very close to the threshold for the onset of the bar-mode instability, nonlinear mode-couplings may be very important in this region and the cause of the observed growths.

The general behaviour of the mode dynamics is reported in figure 5 for the representative model $\mathrm{Sd} 4$, where in the left panel we show the rest-mass isodensity contours in the equatorial plane and at $t=20.8 \mathrm{~ms}$, while in the right one the evolution of the global modes as defined in equation (13) but as measured with respect to the centre of mass of the system (see discussion in section IV of paper I for the definition of the centre of mass in this context). When looking at this panel it is evident that the $m=3$ unstable deformation is the fastest growing one. Furthermore, the $m=2$ does not show any sign of unstable growth, which is instead shown by the $m=1$ deformation. Such growth starts roughly at the time when the $m=1$ and $m=2$ modes have powers comparable with the background $m=4$, i.e. at $t \sim 20 \mathrm{~ms}$, and it continues for the rest of the simulation. The results on the frequencies $f_{3}$ and the growth times $\tau_{3}$ of the $m=3$ instability are collected in table 7 and have been computed using the same analysis discussed for the $m=2$ deformation. It should be noted that the properties of the $m=3$ instability are much more difficult to estimate accurately as the corresponding deformations are smaller than those measured for the bar-mode instability.

It is probably worth stressing that this is the first time that similar instabilities are seen in fully general relativistic simulations of stellar models with a stiff EOS and a moderate degree of differential rotation. In a recent work carried out in Newtonian physics [6], in fact, the development of deformations with $m=3$ was also found but for stellar models with lower values of $\beta$ and with a stronger differential rotation. Furthermore, because the models were studied by imposing an initial artificial $m=2$ perturbation that could alter the global dynamics, it is indeed difficult to understand if the origin of this unstable deformation is purely dynamical or triggered by a nonlinear coupling of modes. Nevertheless, it is interesting to note that in all the simulations reported in [6] as well as in those presented here the $m=1$ 
deformation becomes the dominant one in the final stages of the evolution. This lends support to the idea that the suppression of non-axisymmetric deformations over a dynamical timescale is a generic feature of these instabilities for isolated stars and not necessarily restricted to stellar models with high values of $\beta$.

At the moment and besides the classical $m=2$ bar-mode instability, a proper understanding of the conditions that lead to the development of dynamical instabilities is still lacking. The first attempts at interpreting these instabilities, and especially the low- $\beta$ $m=1$ instability, have been made recently by several authors $[6,16]$. However, further work is needed to clarify the role of $\beta$, of the differential rotation law, of the EOS and of the compactness in determining the growth times, the maximum amplitudes and the persistence of these unstable deformations. Most importantly, work will be needed to finally reach sufficient conditions for the onset of these instabilities whose development has been revealed by numerical simulations.

\section{Conclusions}

We have presented accurate simulations of the bar-mode dynamical instability in full general relativity. The main motivation behind this work was to address some important open questions about the nonlinear features of non-axisymmetric dynamical instabilities in rapidly rotating compact stars. The most important among these questions, because of the impact it has on the global detectability of these stars as sources of gravitational waves, is the determination of the timescale over which the non-axisymmetric deformations persist once these are fully developed.

In order to reach a better understanding of the physics governing dynamical instabilities, we have analysed the onset and development of the bar-mode instability for a large number of stellar models spanning a wide range of masses and angular momenta. The initial models have been calculated as stationary equilibrium solutions for axisymmetric and rapidly rotating relativistic stars modelled as polytropes with adiabatic index $\Gamma=2$ and polytropic constant $K=100$. All the stars have been constructed with a differential-rotation profile having $\hat{A}=1$ and as members of four sequences of constant rest-mass, with $M_{*}=1 M_{\odot}, 1.51 M_{\odot}, 2 M_{\odot}$ and $2.5 M_{\odot}$, respectively. This large set of initial data containing a total of 59 models has allowed us to confirm and extend the results presented in paper I. More precisely, we have analysed the effects that the stellar compactness has on the threshold for the onset of the dynamical bar-mode instability as well as of other dynamical instabilities. Moreover, using an extrapolation technique developed and tested in paper I, we have determined the threshold with great accuracy and for a wide range of compactness, finding good agreement with the Newtonian prediction and improving the previous PN estimates made in [14].

While studying the dynamics of models with $M_{*} \geqslant 2 M_{\odot}$ and values of $\beta$ near the threshold for the development of the bar-mode instability, we have also found stellar models that show the development of a dynamical instability with a dominant $m=3$ deformation, without any seed perturbation of that type. The appearance of these instabilities, whose growth time and frequency have been computed using the same methodology developed for the bar-mode instability, may be rather generic in stars with high mass and deserve additional attention.

Finally, we remark that for all the simulated models, the deformations generated by the non-axisymmetric instabilities are suppressed over a dynamical timescale either as a result of nonlinear mode-couplings or as a result of the conversion of the excess rotational kinetic energy into internal energy. In all cases we have observed the emergence of a residual $m=1$ deformation in the final stages of the instability and before an axisymmetric configuration 
is recovered. These results confirm our previous findings presented in paper I and are in agreement with those recently reported in [6], thus lending support to the idea that the suppression of non-axisymmetric deformations over a dynamical timescale is a generic feature in isolated stars. Further work is clearly needed to confirm or reject this conjecture and to derive the sufficient conditions for the onset of these 'odd- $m$ ' instabilities.

\section{Acknowledgments}

It is a pleasure to thank Harald Dimmelmeier and Ian Hawke for useful discussions and suggestions. GMM is also grateful to Jan Christian Bryne and the Bergen Center for Computational Science for the kind hospitality. This work used the Cactus computational toolkit [17] and the Ccatie code for the solution of the Einstein equations [18]. Support for this research comes also through the SFB-TR7 of the German DFG and through the OG51 of the Italian INFN. GMM is presently a 'Dalla Riccia' fellow at the MPA. All the computations were performed on the cluster for numerical relativity 'Albert' at the University of Parma and 'Peyote' at the AEI.

\section{References}

[1] Baiotti L, De Pietri R, Manca G M and Rezzolla L 2007 Phys. Rev. D 75044023

[2] Shibata M and Sekiguchi Y 2005 Phys. Rev. D 71024014

Saijo M 2005 Phys. Rev. D 71104038

Ott C D, Ou S, Tohline J E and Burrows A 2005 Astrophys. J. 625 L119-22

Ott C D et al 2007 3D collapse of rotating stellar iron cores in general relativity with microphysics Class. Quantum Grav. 24 S139 (Preprint astro-ph/0609819)

[3] Dessart L, Burrows A, Ott C D, Livne E, Yoon S C and Langer N 2006 Astrophys. J. 6441063

[4] Shibata M, Taniguchi K and Uryu K 2003 Phys. Rev. D 68084020

Shibata M, Taniguchi K and Uryu K 2005 Phys. Rev. D 71084021

Shibata M and Taniguchi K 2006 Phys. Rev. D 73064027

Oechslin R, Janka H T and Marak A 2006 Relativistic neutron star merger simulations with non-zero temperature equations of state: I. Variation of binary parameters and equation of state Preprint astro-ph/0611047 (Astron. Astrophys. to be published)

Oechslin R and Janka H T 2007 Gravitational waves from relativistic neutron star mergers with nonzerotemperature equations of state Preprint astro-ph/0702228

[5] Bonaldi M et al 2003 Phys. Rev. D 68102004

Bonaldi Met al 2006 Phys. Rev. D 74022003

[6] Ou S and Tohline J E 2006 Astrophys. J. 6511068

[7] Stergioulas N and Friedman J L 1995 Astrophys. J. 444306

[8] Richtmyer R D and Morton K W 1967 Difference Methods for Initial Value Problems (New York: Interscience)

[9] Nakamura T, Oohara K and Kojima Y 1987 Prog. Theor. Phys. Suppl. 901

[10] Baiotti L, Hawke I, Montero P and Rezzolla L 2003 A new three-dimensional general-relativistic hydrodynamics code Computational Astrophysics in Italy: Methods and Tools, Vol 1, Mem. Soc. Astron. It. Suppl. (Trieste) ed R Capuzzo-Dolcetta p 327

Baiotti L et al 2005 Phys. Rev. D 71024035

[11] Chandrasekhar S 1969 Ellipsoidal Figures of Equilibrium (New Haven, CT: Yale University Press)

[12] Lomb N R 1976 Astrophys. Space Sci. 39 447-62

[13] Karino Sand Eriguchi Y 2003 Astrophys. J. 592 1119-23

[14] Saijo M, Shibata M, Baumgarte T W and Shapiro S L 2001 Astrophys. J. 548 919-31

[15] Rampp M, Mueller E and Ruffert M 1998 Astron. Astrophys. 332969

[16] Watts A L, Andersson N and Jones D I 2005 Astrophys. J. 618 L37 Saijo M and Yoshida S 2006 Mon. Not. R. Astron. Soc. 368 1429-42

[17] www.cactuscode.org

[18] Alcubierre M, Brügmann B, Dramlitsch T, Font J A, Papadopoulos P, Seidel E, Stergioulas N and Takahashi R 2000 Phys. Rev. D 62044034 